\title{
Emergency Care Management with Location-Aware Services
}

\author{
Shih-wei Lee* ${ }^{*}$ Shao-you Cheng*, Jane Yung-jen Hsu*, Polly Huang ${ }^{\dagger}$, Chuang-wen You* \\ * Department of Computer Science and Information Engineering \\ National Taiwan University, Taiwan \\ \{r94094, r93070, yjhsu, f91023\}@Csie.ntu.edu.tw \\ $\dagger$ Department of Electrical Engineering, National Taiwan University, Taiwan \\ phuang@cc.ee.ntu.edu.tw
}

\begin{abstract}
As indoor localization technologies become more affordable and sophisticated. An emerging market has yet to be realized commercially in the indoor environment. We think that the first adopter will arise from the healthcare domain where the need is the most critical. Thus, We aim to implement a set of location-aware services to assist in the management of the emergency department. We apply NTU Taroko, an active RFID module for to real time location tracking on patients, hospital assets, and medical staffs. In addition, we integrate context-aware system proactively to infer event notifications for reminding physicians and nurses. With the proposed system, we can shorten the process of emergency visit effectively and improve the quality of emergency care.
\end{abstract}

\section{INTRODUCTION}

Outdoor localization systems such as Global Positioning System (GPS) have enabled a number of commercial location-aware services, for example, the vehicular navigation systems that are routinely used by many drivers today. An emerging market that has yet to be realized commercially is at the location-aware services for indoor environment. As indoor localization technologies become more affordable and sophisticated, locationaware services for indoor environments are of great commercialization potential. To some degree, commercial applications such as building security and asset tracking fall also into the location-aware service category.

While many of the indoor location-aware applications have been prototyped and proven useful. There are many indoor positioning solutions available on the market, such like Ekahau [1], Sonitor [2], or Versus [3], etc. These companies apply WiFi, ultrasound/RFID, or infrared/RFID technologies in real time locating systems and have successfully deployed these systems in the hospital environment. We think that similar locationaware applications will become more and more popular in Taiwan, and the first adopter, the first real everyday use, will arise from the healthcare domain where the need is the most critical. Therefore, we try to implement and experiment a set of location-aware services to assist in the management of emergency department (ED). This paper introduces an integrated project that brings together the expertise of the emergency medicine doctors at NTU Hospital, the engineers of IBM Taiwan, and the researchers in NTU iSpace Lab. The three parties support respectively the application, service, and infrastructure aspects of the ER management system.

The statistical data shows that NTU Hospital treats about 250 emergency patients a day. This amount is much more than many other countries. However, only one-fourth require admission or stay for observation. With triage, the critical patients would be served firstly, but most of the non-emergent patients feel dissatisfied after a long-term wait. Half of them want to be treated in ten minutes, only $11 \%$ could accept 30 minutes wait [4]. Because of patients are many and their expected waiting time is short, how to speed up the process of emergency visit becomes primary subject to improve the patients' satisfaction. We learn from doctor's description that nursing staff often spend much time in finding patients, hospital assets (e.g. sickbeds, pumps, or wheelchairs). If we can track their position in the ED, then we can spend less time to find particular patients and have better utilization of medical resources. This also means we shorten the process of emergency visit. In addition, patient and staff tracking enable the chances of providing various of location-aware services to improve the qualities of emergency care. This is the main idea for us to construct a location-aware ED management system.

\section{SCENARIO}

We firstly follow the common process of emergency visit at NTU Hospital, as depicted in Fig. 1, to give an emergency care scenario with assists of the locationaware services. 


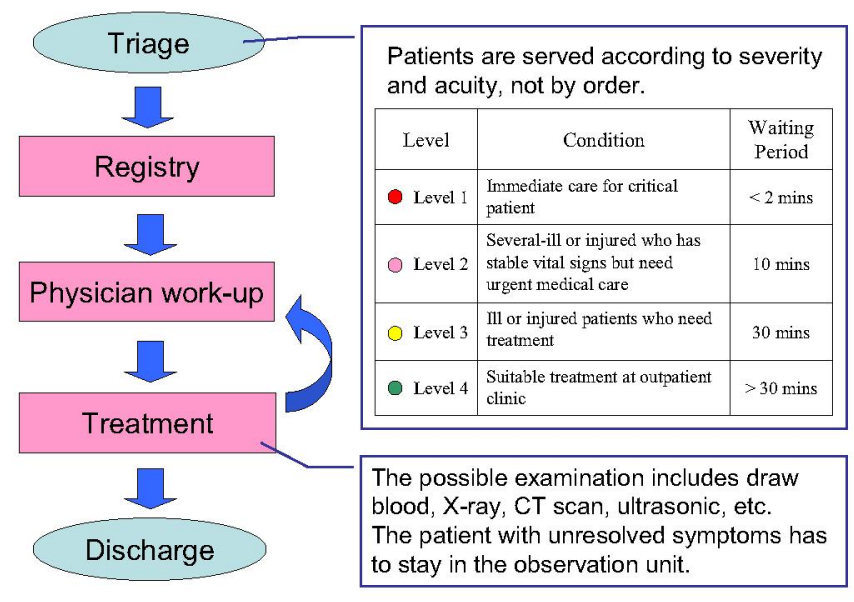

Fig. 1. The process of emergency visit at NTU Hospital

"Bob was involved in a car accident and hurt his legs. He was sent to NTU Hospital by the ambulance. Upon arriving at the hospital, Bob was classified as a class-3 urgent patient and tagged with an active RFID badge. Polly, a murse, used the location service to locate Jane, the on-call doctor who was taking care of other patients in the observation unit, and informed her that a patient just registered and needed care. Without any delay, Jane examines Bob, and orders for an X-Ray to be taken. In the meantime, Polly retrieves the nearest wheelchair available with the help of the location system. After the $X$-Ray, Polly settles Bob straight on a spare bed nearby while waiting for the X-Ray outcome. Twenty minutes later, the management system notifies Jane as soon as Bob's X-Ray becomes available. Jane diagnoses that Bob has a fissure fracture based on the X-Ray result. $B o b$ is immediately located and treated with stabilizing casts on his legs. After paying the medical expenses and having the RFID badge untagged, Bob is released from the hospital."

As can be demonstrated in this scenario, the overhead (tagging and untagging the patient) of using the system is minimal compared with the total amount of time for the nurses to look for various things and for the doctor to discover test results. The efficiency of emergency care and flow can be greatly improved by eliminating such unnecessary delays with the location-aware services.

\section{Emergency CARE Management System}

To enable the improved scenario, the proposed ER management system can be viewed conceptually as an integration of two systems, the location system and the context-aware system. Fig. 2 illustrates the deployment

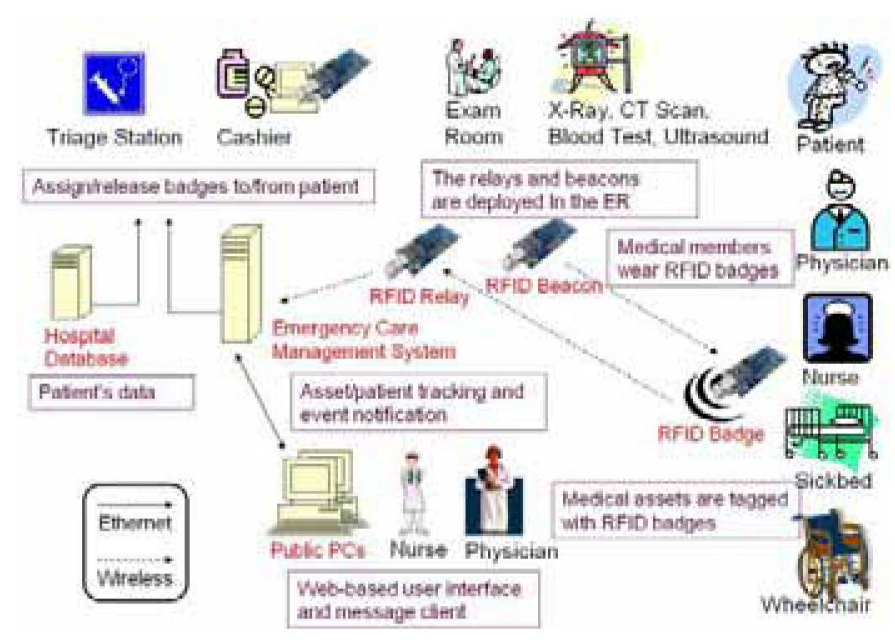

Fig. 2. Deployment of the location system in the ED

of location system in the ED at NTU Hospital. In our current prototype, the location system supports location tracking on hospital assets, medical staffs, and patients. The tracked assets include sickbeds and wheelchairs. They are tagged with active RFID modules for RF-based localization. The physicians and nurses also wear active RFID badges for their shift or visit. Each patient would be assigned an active RFID badge after admitting, the badge has ID number that is associated to the patient. The emergency physicians and nurses can also use their badges as identification to log into the emergency care management system through web-based interface. After successful login, the medical staffs can access location information according to their roles or shifts.

The RFID badges basically are RF receivers that periodically listen for the signals from the RF transceiver, called beacon. After collecting the signals, the badge sends the data to another RF receiver, called relay. The relays further forward the data through sensor network infrastructure to the location engine which determines the badges' position. More detailed descriptions about how to compute a badge's position is given in Section IVB.

Context is the information that can help computer systems to characterize the user's situation, such as the user's physical, social, and emotional information. The main context is often referred to the user's location, activity, identity, and time [5]. A system is context-aware if it uses context to provide relevant information and services to the user. In our ED management system, we develop several emergency care services based on the context, especially location context, to improve patient care. 
We acquire location context by utilizing the location system, however, we are not limited to use single type of context. We have considered other useful contexts, e.g. time, patient's treatment status, staff's activities, test progress, etc. In Section III-A, we introduce three common problems in the ED and respectively develop their context-aware solutions.

\section{A. Emergency Care Services}

First, "uninformed discharges" usually troubles the medical staffs, i.e. a patient who leaves ED prematurely without completing the regular ER procedures. The main reason is due to long-term wait [6]. To manage such accidental situations, we develop a location-aware notification service to support detection of uninformed discharges. Our system continuously monitor patients' location and their treatment status, once such situation occurs, the notification will immediately inform the medical staffs.

Second, the emergency patients expect to be treated as soon as possible, but most of them are not emergent cases. To control their staying time clearly, the system continuously monitors patients' room-level location in the ED and also their treatment status to estimate their waiting time. If there are any patient who has long-term staying without taking any medical service, their location will be displayed on the map to remind the emergency nurses. Moreover, as the study suggests [7], the more responses and concerns the medical staffs give, the more satisfaction the patients feel. Based on this idea, we develop a time-based notification that proactively informs the medical staffs when there are patients' waiting time exceeding 30 minutes.

Third, additional clinical examination is quite often in the emergency visit, however, such test often results in some delays. For example, a patient who needs to take X-Ray, but can not find where to take it. Maybe the patient completes the examination, but he does not know where to go next, etc. There are many possible situations, however, waiting for final results is a common delay. Therefore, we develop an event notification service that proactively informs the physician or nurses when particular patient's X-Ray result is available. The notification has two ways, one is flashing badge's LED light, and the other is sending text messages to the webbased notification board.

The notifications described above are triggered by reasoning about different contexts, including patient's treatment status, patient's location history, physician's

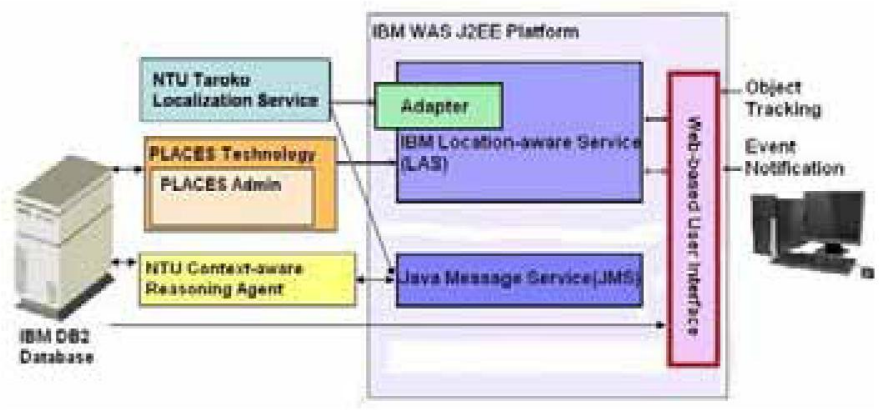

Fig. 3. Main components of the proposed emergency care management system

treatment instructions, software events from HIS $^{1}$ and $\mathrm{PACS}^{2}$, and temporal information.

\section{SYSTEM COMPONENTS}

The proposed emergency care management system is composed by five components, including hardware and software. As Fig. 3 shows, these components are: (1) active RFID module, (2) NTU Taroko Localization Service, (3) IBM Enterprise Location Aware Service Technology, (4) Context-aware Reasoning Agent, and (5) Web-based User Interface. We further describe each component's technical details as follows.

\section{A. Active RFID Module}

The passive RFID technology allows identification of objects and persons in short sensing range. Limited by the radio range, the passive RFID technology can not support indoor localization in our emergency care applications. Therefore, we choose active RFID to enable both identification and localization for the location-aware services.

Taroko is a ultra low power, low cost, wireless embedded device. The module consists of a TI MSP430 microcontroller, an IEEE 802.15.4/Zigbee radio, and $1024 \mathrm{kB}$ size external flash memory. The MSP430 microcontroller operates at $8 \mathrm{MHz}$ and has $10 \mathrm{kB}$ RAM. The Chipcon CC2420 Radio Transceiver operated at $2.4 \mathrm{GHz}$ with $250 \mathrm{kbps}$ data rate and is compliant with IEEE 802.15.4/Zigbee. The flash memory allows developers to store data such as the identification or radio signal strength for location computation. Data collection and communication to PC are via standard USB interface. Taroko also supports integrating with additional sensors by two onboard expansion connectors.

\footnotetext{
${ }^{1}$ Hospital Information System

${ }^{2}$ Picture Archiving and Communication System
} 


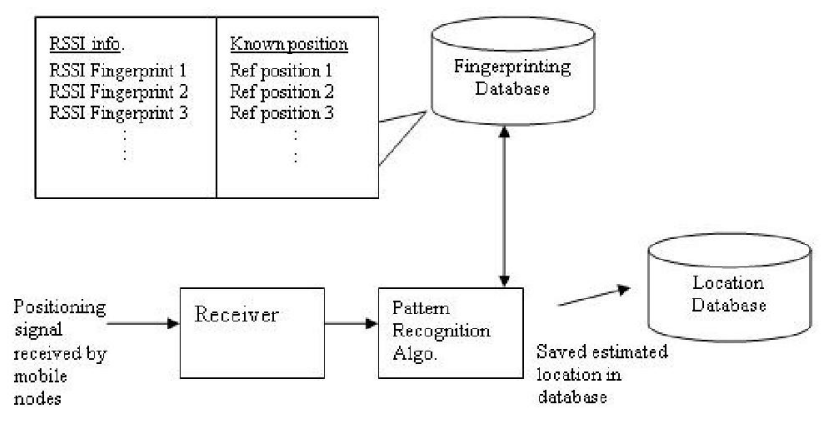

Fig. 4. RF-based location system with fingerprinting approach

Taroko is a programmable module. Developers can code in NesC programming language and run the program upon TinyOS [8], a small and open-source software operating system designed for sensor networks. Alternatively, the developer can choose to program in $\mathrm{C}$ and compile the code by commercial compilers (e.g. IAR embedded workbench) or GNU MSPGCC compiler for the MSP430 microcontroller. The compiled machine code can be uploaded to Taroko via USB port.

\section{B. NTU Taroko Localization Service}

The localization service [9] takes the fingerprinting approach and works in two phases. The first phase is called offline training phase in which a human operator performs a site survey by measuring the received signal strength indicator (RSSI) from different beacon nodes (BNs) at some fixed sampling points in the environment. Given these RSSI measurements, one can compose a radio fingerprint of $\mathrm{BNs}$ at different sampling points. These radio fingerprints will be stored in the Fingerprinting Database. The second phase is known as online estimation phase. Each mobile node is carried by a person or attached to an object, it gathers the RSSI values from BNs and sends the data back through beacon nodes or other relay nodes to a backend location server. Finally, the location of mobile node is determined by matching the measured RSSI values to the stored radio fingerprints. The matching process is computed by applying a pattern recognition algorithm. The location server continuously computes the location of mobile nodes. Fig. 4 shows the main elements in the RF-based location system.

We have deployed a number of Tarokos as BNs in both NTU CSIE building and IBM China Software Development Lab (CSDL) as our experiment environments. The BNs are placed approximately six meters apart throughout the ceilings and are hardwired to the building's power source. The tracked person wears Taroko

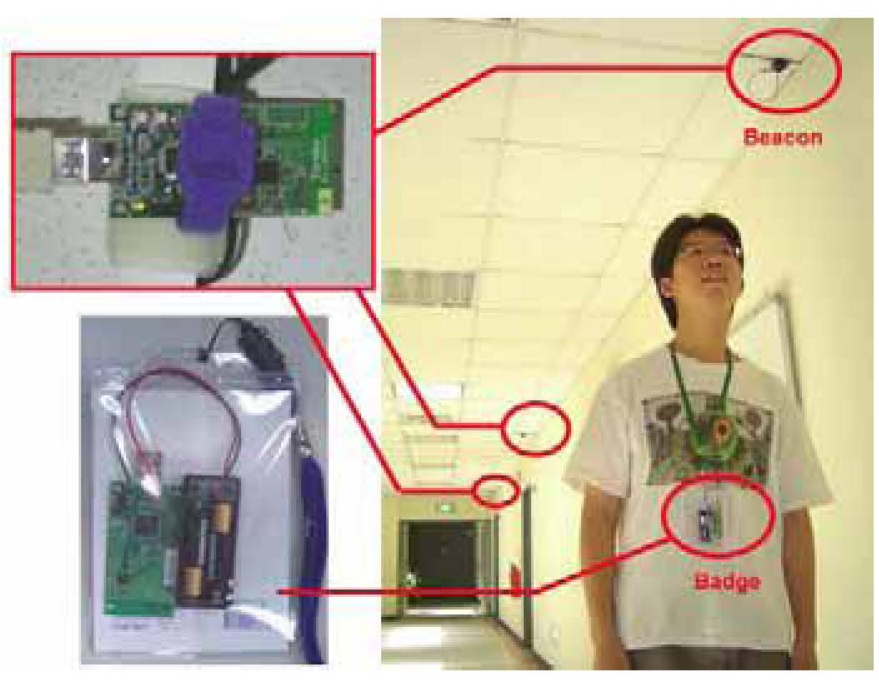

Fig. 5. Indoor deployment of Taroko-based location system; beacons and relays are attached to ceilings in passages, and a badge is worn by a person

as badge. Fig. 5 depicts the environment setting. The badge regularly collects beacon messages and forwards the average RSSI values to the location server to compute its location. In our preliminary experiments, the average accuracy is 1 to 2 meters. To compare Tarokobased location system with traditional WiFi-based (using 802.11 standard) location system. The former has some advantages, including inexpensive RFID module (about 60 USD per Taroko), small (about the size of a cigarettecase), low-power (powered by two AAA batteries), and easily programmable (programming and communicating via USB port).

\section{IBM Enterprise Location Aware Service Technology}

IBM Enterprise Location Aware Service (LAS) [10] is a software middleware that leverages IBM Watson Research Center's PLACES (Points-of-interest, Locations, and Asset Catalog for Enterprise Services) Technology. It provides a set of Java APIs to compatibly implement certain core services of OpenGIS Location Service (OpenLS) implementation specification [11], such as Presentation Service (map portrayal), Location Utility Service (a reverse geocoder to transform a geometry position to particular place). IBM Enterprise LAS aims to help developers build their location tracking web applications effortlessly and consolidate different positioning technologies (e.g. RFID, Zigbee, or UWB) seamlessly with an unified interface.

IBM PlacesAdmin is a web-based administration console that tightly cooperates with IBM Enterprise LAS as the backend middleware. PlacesAdmin helps developers 
manage various spatial entities belonging to different layers in a single map. The preferred map is in scalable vector graphics (SVG) format. SVG is a XML markup language for describing two-dimensional vector graphics, both static and animated. By using PlacesAdmin, we import SVG map into the database and create Coordinate Reference System (CRS) for the map. Then, we start to create various spatial entities to represent real world objects in different layers, like rooms, hospital assets, and people, etc.

We develop a dynamic web site with ED's map as our main user interface. In addition, we use Ajax technique to enhance our web application. Because of the requirement for frequently updating the position of mobile nodes, the Ajax-enhanced web page allows users to select multiple pre-defined objects on the single map and render the map without heavy page-reloading. It is especially useful when performing user's query, since the query results are expected to display continuously snd smoothly on the map. By utilizing SVG and Ajax techniques, our web application presents objects' location in a more dynamic manner and greatly avoids the reloading delay resulted from re-generating the whole map.

\section{Context-aware Reasoning Agent}

The context-aware system should automatically provide relevant services at right time and in right place. To infer appropriated actions in the right situation, we develop a software agent that makes context-aware reasoning. The agent performs reactive rule-based reasoning by utilizing the Context-Aware Rule Engine (CARE) [12] as its knowledge base (KB) to deduce actions. Rulebased programming gives developers the flexibility and maintainability to manage the system's behaviors by loading different rule sets and facts. In general, we build CARE as the context-sensitive version of Jess [13], a general purpose rule engine purely developed by Java language. Because of context rapidly changes, CARE asserts context as dynamic facts and maintains them by keeping context updates from the sensors (e.g. RFID).

When context information changes, it will be coded and sent to the reasoning agent to update its KB. For example, if a patient moves from hallway to the adult medicine area in the ED, such room-entrance event will be detected by the location system. The agent continuously receives such context-changing events, consequently updates the patient's location context in the $\mathrm{KB}$, and finally triggers certain event notifications by rule-based inference. Since we have many distributed

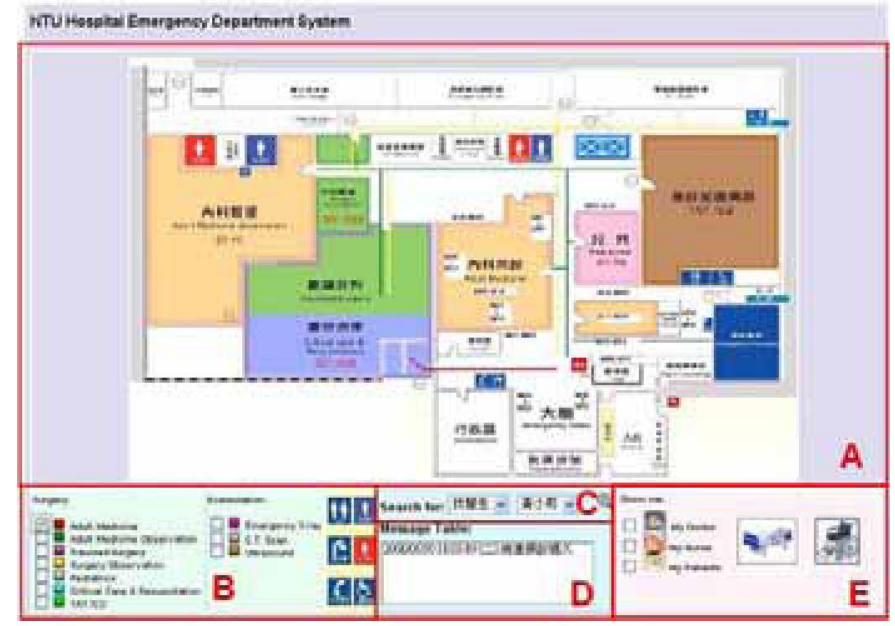

Fig. 6. Web-based user interface for the location-aware emergency care

software components to support different functionalities in our emergency care management system. We apply event-driven architecture design based on Java Message Service (JMS) to facilitate interoperability between these components. JMS supports publish/describe mechanism for the reasoning agent to subscribe the updated context and relevant events from HIS or PACS. These environmental events enrich the agent's KB for performing context-aware reasoning.

So far, we implement the inferred actions by publishing messages to any interested web-based clients in the ED over JMS. For example, if the physician wants to be notified after his cared patients takes X-Ray. After he subscribes to the notification under this particular situation defined in rules, the reasoning agent starts to monitor location context when the patient leaves for examination, and finally infers the notification while the patient finishes the examination and exits the X-Ray area.

\section{E. Web-based User Interface}

We design a web-based user interface to display location information and to provide a query interface for users to search for person and medical resource. The main searching page can be divided into five parts, as Fig. 6 depicts, each part is labeled in capital. Next, we describe each part and its main functionalities as follows.

$\mathrm{A}$ is a map that shows whole emergency care areas in the ED. After user selects some items in part B, C, and $\mathrm{E}$, the query results will be located on the map.

B contains several checkboxes related to the major areas in the ED. The users can click multiple ones to indicate their interesting areas. This part 
also displays important signs, including restrooms, public telephones, convenient stores, etc.

$\mathrm{C}$ is a specialized search bar for looking for specific medical staff in the ED. In the current prototype, we supports searching for physician, nurse, and patient. If the person's role has been selected, the second combo box will retrieve their names from the hospital database and list these names for further selection.

$\mathrm{D}$ is an event notification board that automatically shows the unread notification messages of present login user.

$E$ is a context-aware panel to search for user related information. For example, if a physician logins, it allows the physician to select his cared patients, collaborated nurses, and available assets

\section{CONCLUSION AND Future WORK}

In this paper, we introduce the emergency care management system with location-aware services to improve patient care and emergency visit flow. Based on the RF-based indoor location system, we support patient tracking, staff tracking, and asset tracking. The delay can be reduced by proactive context-aware notifications. We develop an agent-based context-aware system to infer appropriate notifications and apply event-driven design to better integrate these multi-functional components.

Up until now, our system has been successfully deployed and experimented in NTU CSIE building and IBM CSDL to fully test its functionality. To avoid bothering the emergency patients, we are working on the details with administers of Department of Emergency Medicine of NTU Hospital to find out an appropriate area for real deployment and evaluations.

The current prototype system deals with simple emergency care scenarios to demonstrate the benefits of the location-aware services. Our future work includes exploring other complex scenarios for real problems in the ED and evaluating how much improvement we may achieve with the emergency care services. Thought we incorporate a location-aware middleware into the system, we still need more experiences to fully understand its advantages and supports of building location-aware applications.

\section{ACKNOWLEDGEMENTS}

This research was supported by IBM Taiwan and by a grant from the Advanced eCommerce Institute of the Institute for Information Industry (III) in Taiwan. We would like to thank IBM China Software Development
Lab (CSDL) in Taiwan for their technical support of IBM Enterprise LAS and PlacesAdmin. We would like to thank other members of iSpace Laboratory, especially Prof. Polly Huang, Prof. Hao-hua Chu, and Chuang-wen You for their discussion and support of NTU Taroko location system. Special thanks should go to the professionals of the Department of Emergency Medicine of NTU Hospital, Hui-Min Chen and Ken-Shiao Lin for their valuable suggestions.

\section{REFERENCES}

[1] Ekahau. [Online]. Available: http://www.ekahau.com

[2] Sonitor. [Online]. Available: http://www. sonitor.com

[3] Versus. [Online]. Available: http://www.versustech.com

[4] M. H. Chen and Y. C. Huang, "Public awareness of triage and waiting time at emergency department," Journal of Taiwan Emergency Medicine, vol. 5, no. 3, pp. 128-131, September 2003.

[5] A. K. Dey and G. D. Abowd, "Towards a better understanding of context and context-awareness," Georgia Institute of Technology, College of Computing, Tech. Rep. GIT-GVU-99-22, June 1999.

[6] S. J. Liaw, P. M. Hu, and H. C. Liao, "Patients who leave emergency departments prematurely," Journal of Taiwan Emergency Medicine, vol. 4, no. 2, pp. 40-50, June 2002.

[7] M. L. Liou, "Perceived waiting times, actual waiting times and their relations to satisfaction of patients of the emergency department in a medical center," Master's thesis, Graduate Institute of Health Care Organization Administration, NTU, Taiwan, 2001

[8] TinyOS. [Online]. Available: http://www.tinyos.net

[9] C. W. You, Y. C. Chen, H. H. Chu, P. Huang, J. R. Chiang, and S. Y. Lau, "Sensor-enhanced mobility prediction for energyefficient localization," To appeared in IEEE SECON 2006, Reston, VA, USA, September 25-28 2006.

[10] S. C. Shen and Y. C. Chang, "Best practices for location-aware services," Jun 2006. [Online]. Available: http:/www-128.ibm.com/developerworks/edu wi-dw-wi-best-i.html?S_TACT=105AGX05\&S_CMP=HP

[11] The OpenGIS Location Service (OpenLS). [Online]. Available: http://www.opengeospatial.org/standards/olscore

[12] W. R. Jih, J. Y. J. Hsu, C. L. Wu, C. F. Liao, and S. Y. Cheng, "A multi-agent service framework for context-aware elder care," in AAMAS'2006 Workshop on Service-Oriented Computing and Agent-Based Engineering (SOCABE'2006), May 8-12 2006, pp. $61-75$.

[13] E. F. Hill, "Jess, the rule engine for the java platform," Sandia National Laboratories. [Online]. Available: http: //herzberg.ca.sandia.gov/jess 